'División de Pediatría, Pontificia Universidad Católica de Chile, Santiago, Chile. ${ }^{2}$ Departamento de Salud Pública y Epidemiología. Universidad de los Andes. Santiago, Chile. 3División de Salud Pública, Pontificia Universidad Católica de Chile, Santiago, Chile. ${ }^{4}$ Departamento de Pediatría. Universidad de los Andes. Santiago, Chile aEstadístico, PhD bEstadístico, MSc.

Los autores declaran no tener conflictos de interés.

Este proyecto fue financiado por: Universidad de los Andes: Proyecto Med 4, 2006

Recibido el 17 de diciembre de 2013, aceptado el 12 mayo de

Correspondencia a: Dr. Hernán García B. Departamento de Pediatría.

Unidad de Endocrinología Pediátrica. Pontificia Universidad Católica de Chile. Lira $85,5^{\circ}$ piso,

Santiago

Código Postal: 8330074 Teléfono (562)23548084. Fax: 56-2-26384307 hgarciab@med.puc.cl

\section{La razón cintura estatura en escolares no varía con el género, la edad ni la maduración puberal}

\author{
PILAR ARNAIZ ${ }^{1}$, FRANCISCA GROB ${ }^{1}$, GABRIEL CAVADA ${ }^{2, a}$, \\ ANGÉLICA DOMÍNGUEZ ${ }^{3, b}$, RODRIGO BANCALARI ${ }^{1}$, \\ VERÓNICA CERDA ${ }^{4}$, JUANITA ZAMORANO ${ }^{4}$, \\ MANUEL FERNÁNDEZ ${ }^{4}$, HERNÁN GARCÍA ${ }^{1}$
}

\section{Waist-to-height ratio does not change with gender, age and pubertal stage in elementary school children}

Background: Waist-to-height ratio (WHtR) is a cardiometabolic risk indicator in children. A value greater than or equal to 0.55 is an effective screening tool for identifying obese children with metabolic syndrome. However, it is unclear whether this cutoff can be applied equally to any age or gender. Aim: To analyze the variability of WHtR by age, gender and pubertal stage in elementary school children. Patients and Methods: Cross-sectional study in 2,980 school children (6-14 years old, 51\% male) of Santiago, Chile. We measured weight, height and waist circumference and calculated body mass index and WHtR. Pubertal stage was assessed and classified as peripubertal (Tanner I and II) and pubertal (Tanner III, IV and V). Results: The mean age was $9.9 \pm 2.3$ years, with no gender difference $(p=0.5)$. Eighty one percent of boys and $59.4 \%$ of girls were peripubertal $(p<0.001)$. The association between age-adjusted WHtR by gender and pubertal stage was not significant $(p=0.409)$. Therefore mean, standard deviation and percentiles of WHtR were calculated without sex and pubertal stage segmentations. Conclusions: Since WHtR does not vary with age, gender and pubertal status in elementary school children, it is possible to use a single cutoff value, previously defined in this population, to identify children with cardiometabolic risk.

(Rev Med Chile 2014; 142: 574-578)

Key words: Adolescent; Child; Metabolic Syndrome X; Obesity, abdominal.
E 1 sobrepeso y la obesidad en los niños están asociados con un aumento de la prevalencia de numerosos factores de riesgo cardiometabólicos ${ }^{1}$. Una publicación reciente en 3.325 escolares chilenos de educación básica mostró una prevalencia de exceso de peso de $37 \%$ y una clara asociación entre exceso ponderal, resistencia a la insulina y presencia de síndrome metabólico (SM) y sus componentes ${ }^{2}$.

El exceso de grasa central (intra-abdominal) en los niños está relacionada con el desarrollo de enfermedad cardiovascular y diabetes tipo 2 en la edad adulta ${ }^{3}$. La razón cintura estatura (RCE) (perímetro de cintura/estatura, ambos en centímetros) es una medida antropométrica que da mejor cuenta de la adiposidad central y, por lo tanto, tiene mejor capacidad de determinar el riesgo cardiometabólico tanto en adultos como en niños y adolescentes ${ }^{4,5}$.

En hombres y mujeres adultos, un valor $\geq 0,50$ es una medida sensible para identificar a este grupo de riesgo ${ }^{6}$. Este valor también sería aplicable en 
pediatría $^{7,8}$. Un estudio que lo aplicó en escolares chilenos demostró que el punto de corte de 0,55 era capaz de detectar con $71,6 \%$ de sensibilidad y $70,2 \%$ de especificidad la presencia de $\mathrm{SM}^{9}$. No sólo los niños obesos sino también los niños con sobrepeso pueden variar en su grasa abdominal y presentar factores de riesgo cardiometabólicos y la RCE también es capaz de especificar el riesgo en este grupo ${ }^{10}$. Sin embargo, se ha sugerido que este punto de corte sería diferente y específico para género, etnia y grado de urbanización, por lo que sería necesario que cada población contara con percentiles de referencia propios ${ }^{11,12}$. Además podría variar en cada grupo etario ${ }^{13}$.

El objetivo de este estudio fue determinar si la RCE varía en escolares chilenos de educación básica, de acuerdo a género, edad y desarrollo puberal.

\section{Pacientes y Métodos}

Se realizó un estudio transversal descrito previamente $^{14}$, en 2.980 escolares de 6 a 14 años, de nivel socioeconómico medio-bajo y bajo, reclutados desde establecimientos municipales subvencionados de la Región Metropolitana de Santiago, Chile, entre marzo y diciembre del año 2006.

\section{Medidas antropométricas}

Se determinó la estatura en posición erecta con un estadiómetro de pared fijo marca SECA ${ }^{\circledR}$. El peso se obtuvo con los niños descalzos y en ropa interior con una pesa marca SECA modelo 700. El perímetro de cintura (PC) se midió con una cinta métrica inextensible, trazando una línea horizontal sobre las crestas ilíacas, paralela a la línea media axilar, luego de que el paciente exhalara normalmente y de acuerdo a las guías de NHANES ${ }^{15}$. Todas las medidas antropométricas se realizaron tres veces y se utilizó un promedio de ellas para el análisis. Se calculó el índice de masa corporal (IMC) dividiendo el peso en kilos por la estatura en metros ${ }^{2}$. El estado nutricional fue clasificado de acuerdo al Centers for Disease Control (CDC $)^{16}$ aplicando percentiles de IMC: normal: 5 a 84 ; sobrepeso: 85 a 94; obeso: $\geq 95$; y bajo peso: $<5$; exceso de peso fue definido como percentil de IMC $\geq 85$. La RCE se obtuvo de la división del perímetro de cintura por la estatura, ambas medidas expresadas en centímetros. Pediatras entrenados evaluaron el estadío puberal de acuerdo a los grados de Tanner ${ }^{17}$, y se clasificaron en dos grupos definidos como peribuberales (mujeres con Tanner I o II de mamas, varones con testes Tanner I o II) y puberales (mujeres con Tanner de mamas mayor o igual a III, varones con testes Tanner mayor a III.

\section{Análisis estadístico}

Las características generales del grupo se resumen como número de casos y porcentaje para las variables categóricas, y como promedio y desviaciones estándar (DE) para las variables numéricas. La RCE se describe según edad, mediante percentiles, media y DE. Para comparaciones entre dos variables categóricas se utilizaron pruebas $\chi^{2}$ de Pearson. La RCE presentó una distribución no normal, por lo que se aplicaron pruebas no paramétricas para contrastar con variables categóricas: Mann-Whitney al asociar con sexo y KruskallWallis al asociar con Tanner. Para evaluar el efecto de la edad en la RCE, ajustado por sexo y Tanner, se usó un modelo de regresión lineal múltiple. El nivel de significancia utilizada fue de 0,05. Los datos fueron procesados en STATA, versión 11.

Este trabajo fue aprobado por el Comité de Ética de la Investigación en Seres Humanos de la Facultad de Medicina de la Universidad de Chile y por el Comité de Ética de la Universidad de los Andes. Se requirió la firma de consentimiento informado del apoderado y el asentimiento del niño mayor de 12 años y la asistencia voluntaria del sujeto.

\section{Resultados}

La edad promedio fue $9,86 \pm 2,33$ años, sin diferencias por sexo $(p=0,531)$. El $81,4 \%$ de los hombres y $59,4 \%$ de las mujeres fueron peripuberales (Tanner I y II) $(\mathrm{p}<0,001)$. La prevalencia de mal nutrición por exceso (sobrepeso más obesidad) fue $45,1 \%$ con mayor porcentaje en hombres $(\mathrm{p}<0,01)$ (Tabla 1).

Los valores de la RCE fueron de similar magnitud y variabilidad, tanto en hombres y mujeres, como a través de las edades (Figura 1). Para el grupo de los hombres, se visualizó un patrón donde, en las edades de 6 y 7 años y de 13 y 14 años la RCE estaría disminuida respecto de las edades centrales, no siendo estas diferencias significativas. Mediante regresión lineal múltiple se asoció la RCE con la 
Tabla 1. Características generales de escolares de 6 a 14 años. Santiago, 2006

\begin{tabular}{|lccc|}
\hline Característica & $\begin{array}{c}\text { Hombre } \\
(\mathbf{n = 1 . 5 3 6 )}\end{array}$ & $\begin{array}{c}\text { Mujer } \\
\text { (n= 1.444) }\end{array}$ & Valor p \\
\hline Edad (años); media (DE) & $9,88(2,31)$ & $9,83(2,36)$ & 0,531 \\
Peripuberal; $n$ (\%) & $280(32,44)$ & $583(67,56)$ & $<0,001$ \\
\hline Puberal; $n$ (\%) & $1.227(58,99)$ & $853(41,01)$ & $<0,01$ \\
\hline Exceso de peso; $n$ (\%) & $733(46,7)$ & $609(42,4)$ & \\
\hline
\end{tabular}

DE: desviación estándar. IMC: Índice de Masa Corporal.

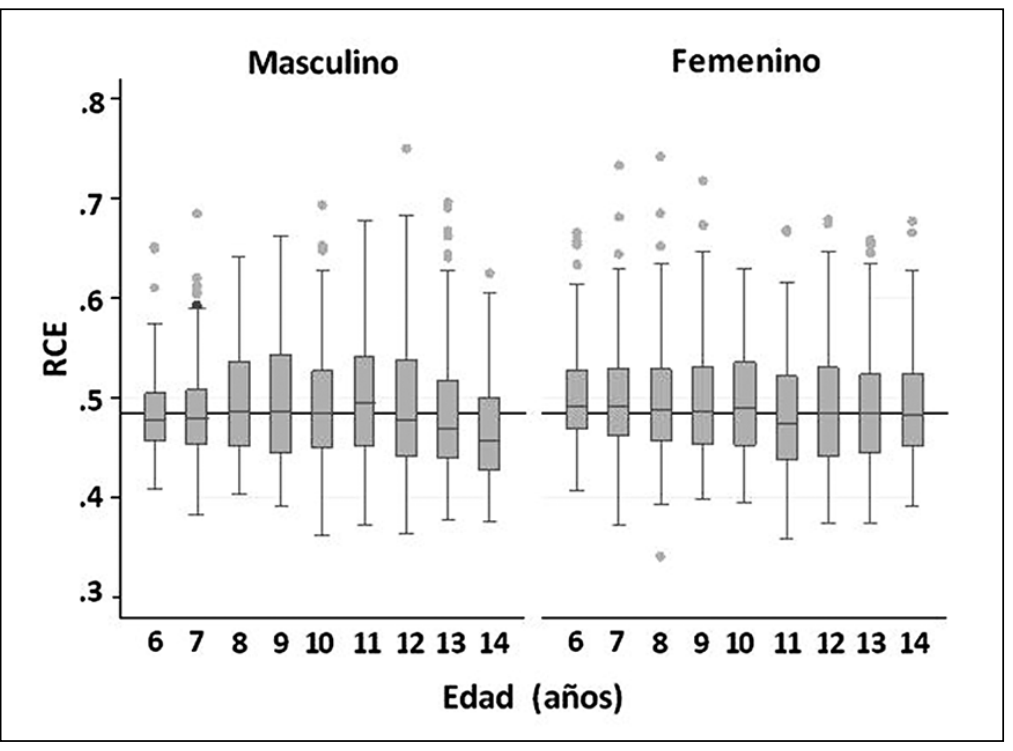

Figura 1. Distribución de los valores de RCE para las diferentes edades, según género, en escolares de 6 a 14 años. Santiago, 2006. Las gráficas de Boxplot muestran que tanto hombres como mujeres de entre 6 y 14 años presentan valores de la RCE similares en magnitud y variabilidad. La línea central de cada caja, muestra la mediana de RCE específica para el sexo y la edad indicada, mientras que la línea horizontal muestra el promedio global de RCE.

edad, ajustada por sexo y maduración puberal lo que no fue significativo $(\mathrm{p}=0,409)$, por lo que se calculó el promedio, DE y los percentiles de la RCE, sin diferenciar por las dos variables de ajuste. La estabilidad de los valores de RCE observada en la Figura 1 se confirma en la Tabla 2, donde los valores medianos y promedios de RCE resultaron similares para todas las edades. La RCE promedio para el grupo total fue de 0,49 $\pm 0,06$.

La Figura 2 muestra la distribución acumulada de la RCE para los dos géneros y todas las edades en su conjunto. Se observa que $15 \%$ de los escolares presenta una RCE superior a 0,55 , punto de corte considerado como de riesgo. Adicionalmente, existe $22,2 \%$ de escolares con RCE entre 0,50 y 0,55 . Finalmente, se deduce que $62,8 \%$ de los escolares tiene una RCE inferior a 0,50. Además, se puede distinguir asimetría en los valores de RCE,

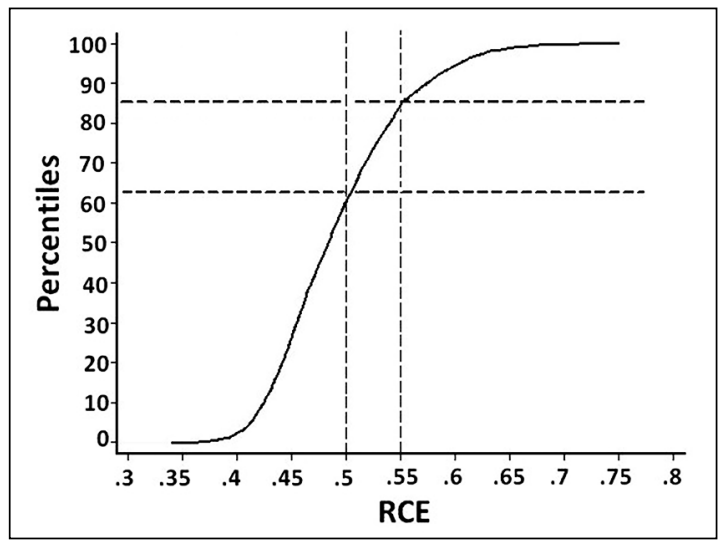

Figura 2. Distribución acumulada de la RCE en escolares de 6 a 14 años. Santiago, 2006. Distribución acumulada de la RCE sin distinguir por sexo o edad. La curva muestra la relación entre los diferentes valores de RCE y el porcentaje de escolares que presentó una RCE menor o igual a dicho valor. 
Tabla 2. Percentiles de RCE por edad en escolares de 6 a 14 años. Santiago, 2006

\begin{tabular}{|c|c|c|c|c|c|c|c|c|c|c|c|}
\hline $\begin{array}{l}\text { Edad } \\
\text { (años) }\end{array}$ & n & Mín & p5 & p10 & p25 & p50 & p75 & p90 & p95 & Máx & $\begin{array}{l}\text { Media } \\
\text { (DE) }\end{array}$ \\
\hline 6 & 263 & 0,41 & 0,43 & 0,44 & 0,46 & 0,49 & 0,52 & 0,55 & 0,58 & 0,67 & $0,49(0,05)$ \\
\hline 7 & 366 & 0,37 & 0,43 & 0,44 & 0,46 & 0,48 & 0,52 & 0,56 & 0,60 & 0,73 & $0,49(0,05)$ \\
\hline 8 & 332 & 0,34 & 0,42 & 0,43 & 0,45 & 0,49 & 0,53 & 0,58 & 0,61 & 0,74 & $0,50(0,06)$ \\
\hline 9 & 364 & 0,39 & 0,41 & 0,43 & 0,45 & 0,49 & 0,54 & 0,58 & 0,60 & 0,72 & $0,50(0,06)$ \\
\hline 10 & 394 & 0,36 & 0,41 & 0,42 & 0,45 & 0,49 & 0,53 & 0,58 & 0,60 & 0,69 & $0,49(0,06)$ \\
\hline 11 & 381 & 0,36 & 0,41 & 0,42 & 0,45 & 0,48 & 0,53 & 0,57 & 0,59 & 0,68 & $0,49(0,06)$ \\
\hline 12 & 398 & 0,36 & 0,40 & 0,42 & 0,44 & 0,48 & 0,53 & 0,58 & 0,61 & 0,75 & $0,49(0,06)$ \\
\hline 13 & 365 & 0,37 & 0,40 & 0,42 & 0,44 & 0,48 & 0,52 & 0,58 & 0,61 & 0,70 & $0,49(0,06)$ \\
\hline 14 & 120 & 0,38 & 0,40 & 0,42 & 0,44 & 0,47 & 0,52 & 0,57 & 0,59 & 0,68 & $0,48(0,06)$ \\
\hline
\end{tabular}

DE: desviación estándar; p: percentil; Mín: mínimo; Máx: máximo.

por lo que la curva muestra una prolongación hacia valores altos de RCE. Esto se condice con la alta presencia de obesidad observada en la muestra estudiada.

\section{Discusión}

La RCE es un indicador antropométrico de obesidad central, de fácil obtención (sólo se requiere de un estadiómetro, una cinta métrica inextensible y un protocolo estandarizado de medición según normas internacionales ya descritas en los métodos). Su valor es relativamente constante a través de diferentes edades, sexos y grupo raciales por lo que sería posible utilizar un punto de corte único de esta razón para todos los niños ${ }^{18,19}$. Sin embargo, otras publicaciones sugieren que esta razón variaría en las edades más extremas como los pre-escolar y adolescentes, recomendando el uso de percentiles ${ }^{20}$. Nuestros resultados confirman que la razón entre estatura y cintura se mantiene constante en el importante grupo etario de escolares de educación básica de ambos sexos y que a estas edades no estaría influenciada por la maduración puberal (Tabla 2 y Figura 1).

La población del estudio presenta un exceso de peso de $45,1 \%$ (Tabla 1), reflejándose en la Figura 2 , la que muestra que $15 \%$ de los escolares presenta una RCE superior a 0,55 , punto de corte considerado como de riesgo para presentar SM. Este dato coincide con lo observado en el SIMCE de Educación Física del 2012 publicado recientemente, en que utilizaron la RCE para detectar riesgo cardiovascular ${ }^{21}$. La misma Figura 2 muestra que $22 \%$ se encuentra en un rango intermedio entre 0,50 y 0,55 . Una publicación en escolares chilenos ${ }^{22}$ mostró que la progresión de la RCE se asoció con la agregación de componentes del SM. Este hecho pone énfasis en que la progresiva sumatoria de componentes del SM, asociado al aumento de la RCE, sería lo más importante desde el punto de vista predictivo para la detección temprana de riesgo cardiometabólico, más que la sola presencia o ausencia del SM (3 o más componentes). Esta idea es confirmada por publicaciones recientes en que, no sólo los obesos tendrían una RCE aumentada sino también algunos normales y con sobrepeso y de ahí la importancia de su medición periódica y derivación oportuna ${ }^{8,10,23}$.

La representatividad de este estudio conforme a su objetivo, está centrada en la suficiente variabilidad de la RCE en cada una de las edades consideradas.

Podemos concluir que el valor de la RCE no varía con la edad, el género ni la maduración puberal en niños entre 6 y 14 años. Estos resultados apoyan el uso de la medición sistemática y a lo largo del tiempo de la RCE por parte de los profesores y pediatras de atención primaria en escolares de educación básica, utilizándolo como un método de tamizaje y seguimiento a lo largo de esta edad escolar, simple y de bajo costo. 


\section{Referencias}

1. Ford ES, Mokdad AH, Ajani UA. Trends in risk factors for cardiovascular disease among children and adolescents in the United States. Pediatrics 2004; 114 (6): 1534-44.

2. Mardones F, Arnaiz P, Barja S, Giadach C, Villarroel L, Domínguez A, et al. Estado nutricional, síndrome metabólico y resistencia a la insulina en niños de Santiago, Chile. Nutr Hosp 2013; 28 (6): 1995-2001.

3. Juonala M, Magnussen CG, Berenson GS, Venn A, Burns TL, Sabin MA, et al. Childhood adiposity, adult adiposity, and cardiovascular risk factors. N Engl J Med 2011; 365 (20): 1876-85.

4. Lee CM, Huxley RR, Wildman RP, Woodward M. Indices of abdominal obesity are better discriminators of cardiovascular risk factors than BMI: a meta-analysis. J Clin Epidemiol 2008; 61: 646-53.

5. Kahn HS, Imperatore G, Cheng YJ. A population-based comparison of BMI percentiles and waist-to-height ratio for identifying cardiovascular risk in youth. J Pediatr 2005; 146 (4): 482-8.

6. Ashwell M, Gunn P, Gibson S. Waist-to-height ratio is a better screening tool than waist circumference and BMI for adult cardiometabolic risk factors: systematic review and meta-analysis. Obes Rev 2012; 13 (3): 275-86.

7. Kuba VM, Leone C, Damiani D. Is waist-to-height ratio a useful indicator of cardio-metabolic risk in 6-10-yearold children? BMC Pediatr 2013; 13: 91.

8. Khoury M, Manlhiot C, McCrindle BW. Role of the waist/height ratio in the cardiometabolic risk assessment of children classified by body mass index. J Am Coll Cardiol 2013; 62 (8): 742-51.

9. Arnaiz P, Acevedo M, Díaz C, Bancalari R, Barja S, Aglony M, Cavada G, García H. Razón cintura estatura como predictor de riesgo cardiometabólico en niños. Rev Chil Cardiol 2010; 29: 281-8.

10. Freedman DS, Dietz WH, Srinivasan SR, Berenson GS. Risk factors and adult body mass index among overweigth children: The Bogalusa Heart Study. Pediatrics 2009; 123: 750-7.

11. Matsha TE, Kengne AP, Yako YY, Hon GM, Hassan MS, Erasmus RT. Optimal waist-to-height ratio values for cardiometabolic risk screening in an ethnically diverse sample of South African urban and rural school boys and girls. PLoS One 2013; 8 (8): e71133.

12. Nambiar S, Hughes I, Davies PS. Developing waist-toheight ratio cut-offs to define overweight and obesity in children and adolescents. Public Health Nutr 2010; 13 (10): 1566-74.

13. Tybor DJ, Lichtenstein AH, Dallal GE, Must A. Waist to height ratio is correlated with height in US children and adolescents aged 2-18 years. Int J Ped Obes 2008; 3: 148-51.

14. Bancalari R, Díaz C, Martínez-Aguayo A, Aglony M, Zamorano J, Cerda V, et al. [Prevalence of hypertension in school age children and its association with obesity]. Rev Med Chile 2011; 139 (7): 872-9.

15. National Health and Nutrition Examination Survey. Anthropometry Procedures Manual, January 2004. Accesado: Mayo 23, 2013. Disponible en: www.cdc.gov/ nchs/data/nhanes/nhanes_03_04/BM.pdf

16. Centers for Disease Control and Prevention. Overview of the CDC Clinical Growth Charts. Accesado: Mayo 23, 2013. Disponible en: www.cdc.gov/nWCdphp/dnBP/ growthcharts/training/modules/module2/text/BPge6b. htm

17. Tanner J. Growth at adolescence, 2nd ed., 1962; Oxford: Blackwell Scientific Publications.

18. Freedman DS, Kahn HS, Mei Z, Grummer-Strawn LM, Dietz WH, Srinivasan SR, Berenson GS. Relation of body mass index and waist-to-height ratio to cardiovascular disease risk factors in children and adolescents: The Bogalusa heart study. Am J Clin Nutr 2007; 86: 3340.

19. Maffeis C, Banzato C, Talamini G. Waist-to-height ratio, a useful index to identify high metabolic risk in overweight children. J Pediatr 2008; 152: 207-13.

20. Roswall J, Bergman S, Almqvist-Tangen G, Alm B, Niklason A, Nierop AFM, Dahlgren J. Population-based waist circumference and waist-to-height ratio reference values in preschool children. Acta Paediatrica 2009; 98: 1632-6.

21. Ministerio de Educación, Chile. Informe técnico Simce 2012. Accesado: Abril 28, 2014. Disponible en: http:// www.agenciaeducacion.cl/simce/informe-tecnico-simce/

22. Arnaiz P, Marín A, Pino F, Barja S, Aglony M, Navarrete $\mathrm{C}$, et al. Índice cintura estatura y agregación de componentes cardiometabólicos en niños y adolescentes de Santiago. Rev Med Chile 2010; 138: 1378-85.

23. Mokha JS, Srinivasan R, DasMhapatra P, Fernández $\mathrm{C}$, Chen $\mathrm{W}, \mathrm{Xu} \mathrm{J}$, et al. Utility of waist-to-height ratio in assessing the status of central obesity and related cardiometabolic risk profile among normal weight and overweight/obese children: The Bogalusa Heart Study. BMC Pediatrics 2010; 10: 73. 